\title{
Capacités de travail du zébu, de l'âne et du cheval au Nord-Cameroun. Concept d'adéquation du couple animal-outil
}

\author{
Éric Vall ${ }^{*}$ \\ Unité de recherche sur l'animal et l'élevage, Cirad-EMVT, BP 5035, \\ 34032 Montpellier, France
}

(Reçu le 8 octobre 1996 ; accepté le 4 novembre 1997)

\begin{abstract}
Working ability of the zebu, the donkey and the horse in northern Cameroon. Concept of animal-tool appropriateness. This study was carried out from 1993 to 1995 in the northern Cameroonian cotton area. This zone was chosen for its representativeness in relation with other situations of the savannah region. A computerised measure system for animal traction to characterise the work efficiency of zebus, donkeys and horses for different draught forces and variable climatic conditions (during dry and wet seasons) was used. The body weights (BW) of donkeys, horses and zebus ranged, respectively, from 100 to $150 \mathrm{~kg}, 225$ to $300 \mathrm{~kg}$, and 325 to $450 \mathrm{~kg}$. The mechanical study shows that the maximal efficiency of work varies for each species, in accordance with different and complementary features. Optimal draught forces determined are about 10 to $16 \% \mathrm{BW}$ for donkeys and horses and 9 to $15 \% \mathrm{BW}$ for zebus. At the optimal draught force level, average speeds of donkeys, horses and zebus are respectively: 3.2 to $2.7 \mathrm{~km} / \mathrm{h}, 3.4$ to $3.3 \mathrm{~km} / \mathrm{h}, 2.8$ to $2.5 \mathrm{~km} / \mathrm{h}$, and the length of work 5.5 to $3.5 \mathrm{~h}$ for donkeys and 6.0 to $4.5 \mathrm{~h}$ for horses and 6.5 to 4.5 for zebus. The energy expenditure link to work is estimated between 5 to $8 \mathrm{MJ}$ for donkeys, 16 to $24 \mathrm{MJ}$ for horses and 24 to $40 \mathrm{MJ}$ for zebus. The mechanical references, built around the concept of animal-tool appropriateness, help in decision making for equipment choice. Some proposals are given to perfect it and include it, in a complete innovation process, starting from prototype material shaping up to the appraisal of the farmer's tools. (@ Elsevier/Inra.)
\end{abstract}

\section{horse / zebu /donkey / draught animal power / northern Cameroon}

Résumé - L'étude relatée a été conduite de 1993 à 1995 dans le bassin cotonnier du NordCameroun. Les capacités de travail du zébu, de l'âne et du cheval ont été caractérisées à l'aide d'une chaîne de mesures informatisée. Les poids vifs (PV) des ânes, des chevaux et des bovins sont respectivement compris entre 100 et $150 \mathrm{~kg}, 225$ et $300 \mathrm{~kg}, 325$ et $450 \mathrm{~kg}$. Les résultats indiquent que les plages des forces de traction optimales sont comprises entre : 10 et $16 \% \mathrm{PV}$ pour les

\footnotetext{
* Correspondance et tirés à part.
}

Tél : 237272024 ; fax : 237272985 
ânes et les chevaux ; 9 et $15 \%$ PV pour les bovins. Entre les bornes de ces plages et selon le poids vif de l'animal : les vitesses moyennes des ânes, des chevaux et des zébus sont respectivement comprises entre 3,2 et $2,7 \mathrm{~km} / \mathrm{h}, 3,4$ et $3,3 \mathrm{~km} / \mathrm{h}, 2,8$ et $2,5 \mathrm{~km} / \mathrm{h}$; les durées de travail entre 5,5 et $3,5 \mathrm{~h}$ chez les ânes, 6,0 et $4,5 \mathrm{~h}$ chez les chevaux, 6,5 et 4,5 h chez les zébus ; les dépenses énergétiques liées au travail entre 5 et $8 \mathrm{MJ}$ chez les ânes, 16 et $24 \mathrm{MJ}$ chez les chevaux et 24 et 40 MJ chez les bovins. L'ensemble des résultats est représenté dans une table des performances à l'effort commune pour les trois espèces qui permet de mettre en relation la dépense énergétique liée au travail, avec la durée et la vitesse de travail, en fonction de la force de traction et du poids vif de l'animal. (@ Elsevier / Inra.)

cheval / zébu / âne / énergie animale / Nord-Cameroun

\section{INTRODUCTION}

Au début des années 1980, il subsistait des lacunes sur la connaissance des performances, la physiologie et l'élevage des animaux de trait [16]. La traction animale en Afrique connaissait des succès divers en raison d'une attention insuffisante accordée aux contraintes liées à l'animal [14]. Depuis, cette connaissance a été approfondie, par des travaux consacrés à l'animal de trait et à la place de la traction animale dans les systèmes de production [2, $4,8,17]$. Par ailleurs, la mise au point de chaînes de mesures informatisées et portables fixées sur des attelages en mouvement a permis un regain de la recherche dans ce domaine $[9,10]$.

En Afrique francophone, les travaux sur les performances des attelages datent des années 1960 [15]. En 1990, le CiradEMVT acquiert une chaîne de mesure. Il choisit d'appliquer cette technologie au Nord-Cameroun. C'est un territoire représentatif de la zone agroécologique de la culture du coton où l'évolution des systèmes de production a été marquée par le développement de la culture attelée : traction bovine dès 1950 , puis asine à partir de 1980 et enfin équine à partir de 1990. En 1995, on dénombrait 37000 paires de bœufs, 14000 ânes et 2000 chevaux. Aujourd'hui, les paysans du Nord-Cameroun confrontés à une crise économique sévère s'orientent vers des attelages peu coûteux tels que les ânes et les chevaux.
Cependant, quelle que soit l'espèce, l'utilisation annuelle des attelages reste faible au plus $30 \mathrm{j}$ [18]. Cette situation pourrait être améliorée par une meilleure maîtrise du travail animal. Le concept d'adéquation animal-outil vise à définir des conditions techniques optimales d'utilisation d'un type d'attelage pour un type d'outil de manière à faciliter le choix d'une formule d'attelage pour un travail requis ou inversement. Cela suppose une connaissance précise des capacités mécaniques des attelages qui actuellement fait défaut.

Cet article présente les résultats d'une étude expérimentale conduite à Garoua de 1993 à 1995. L'objectif est de mettre au point une méthode simple permettant de caractériser les performances à l'effort des trois espèces utilisées localement en fonction de la force de traction et du poids vif de l'animal. Pour chaque espèce, les termes de bilan du travail animal (durée et vitesse moyenne, dépense énergétique) et les plages d'efficacité maximale sont évalués. Les résultats sont reproduits dans une abaque commune aux trois espèces.

\section{MATÉRIEL ET MÉTHODES}

\subsection{Choix des animaux et conduite}

Les caractéristiques des animaux étudiés sont présentées dans le tableau I. L'évolution du poids vif des animaux (PV) est suivie chaque mois. Le nombre d'individus par espèce est faible (5) en raison des contraintes d'utili- 
Tableau I. Description des animaux ayant participé aux essais.

\begin{tabular}{lccc}
\hline Espèces & Bos indicus & Equus asinus & $\begin{array}{c}\text { Equus caballus } \\
\text { Races }\end{array}$ \\
$\begin{array}{l}\text { Goudali (4) } \\
\text { Bororo (1) }\end{array}$ & - & $\begin{array}{c}\text { Barbe (2) } \\
\text { Dongolaw (2) } \\
\text { métisse (1) }\end{array}$ \\
\hline Nombre & 5 & 5 & 5 \\
Sexes & $4 \mathrm{C} ; 1 \mathrm{M}$ & $3 \mathrm{M} ; 2 \mathrm{~F}$ & $5 \mathrm{M}$ \\
Âges en 1993 (ans) & 3 à 6 & 2 à 6 & 5 à 10 \\
Hauteurs au garrot $(\mathrm{cm})$ & 120 à 130 & 95 à 100 & 135 à 145 \\
Poids vifs $(\mathrm{kg})$ & 325 à 450 & 100 à 150 & 225 à 300 \\
\hline
\end{tabular}

$\mathrm{M}$ : mâle ; $\mathrm{C}$ : castré ; F : femelle.

sation de la chaîne de mesure qui ne permettent pas d'observer plus de deux attelages simultanément. Cet inconvénient est contourné en répétant les observations durant 3 années (1993, 1994 et 1995). Les animaux sont maintenus dans un bon état corporel en veillant à leur alimentation (couverture des besoins énergétiques) et à leur santé (déparasitages internes à l'Albendazole en fin saison sèche et en fin de la saison des pluies ; application tous les $15 \mathrm{j}$ d'accaricide à base de Fluméthrine ${ }^{2}$ en saison des pluies). Ce standard est certes éloigné des conditions paysannes, mais il a l'avantage de minimiser les facteurs de confusion qui, conjugués à un effectif limité, auraient exacerbé la variabilité individuelle.

Durant la saison sèche chaude (février à mai), les animaux sont nourris de fourrages secs servis en rations individuelles matin et soir (fanes de légumineuses : arachide ou niébé ; pailles de céréales : maïs, sorgho ou riz). Durant la saison des pluies et la saison sèche froide (juin à janvier), ils sont conduits au pâturage 6 à $8 \mathrm{~h} / \mathrm{j}$. Toute l'année, les bovins reçoivent le matin une ration de tourteau de coton (environ $2 \mathrm{~kg} \mathrm{MS}$ ) et les équidés un mélange ( $50 \%$ maïs ; $50 \%$ tourteau de coton) à raison de 1 et $2 \mathrm{~kg}$ MS respectivement pour les ânes et les chevaux. Tous les animaux sont abreuvés au seau matin et soir en saison sèche et à la rivière en saison des pluies.

\footnotetext{
${ }^{\mathrm{I}} 1$ bolus / prise ; Valbazen n.d.

$225 \mathrm{~mL} /$ application ; Bayticol n.d.
}

\subsection{Chaîne de mesure}

La chaîne de mesure (figure l) se compose d'une centrale d'acquisitions portable (2lx Campbell $\mathrm{Ltd}$ ) reliée à des capteurs placés sur l'attelage, via un boîtier de connexions [5]. Le boîtier permet de piloter la lecture et l'enregistrement des données. Les données enregistrées sont transférables de la centrale d'acquisition vers un module de stockage ( $\mathrm{Sm} 192$ Campbell Ltd). Après ce transfert, elles sont récupérées sur un micro-ordinateur, par l'intermédiaire d'une interface (Sc352 Campbell Ltd), en vue de leur traitement sur un tableur (SuperCalc 5).

La force de traction se mesure par une jauge de contrainte placée sur la ligne de traction (capteur Scc Inox Captels SA). La force oscille irrégulièrement plusieurs fois par seconde, en raison de la marche de l'animal et des frottements de l'outil sur le sol [10]. L'estimation de la force moyenne nécessite un grand nombre de mesures sur une très courte période. C'est pourquoi, un intervalle de $0,2 \mathrm{~s}$ entre deux mesures a été choisi. La centrale d'acquisitions calcule une moyenne arithmétique toutes les 10 mesures (soit toutes les $2 \mathrm{~s}$ ), et stocke cette valeur dans sa mémoire finale.

Les essais sont conduits sur une piste plate rectangulaire d'un périmètre de $1245 \mathrm{~m}$ (sol agricole) ce qui permet de mesurer la distance parcourue en comptabilisant les tours effectués. La vitesse moyenne d'un attelage se calcule par le rapport entre la distance parcourue et le temps écoulé pour parcourir cette distance. 


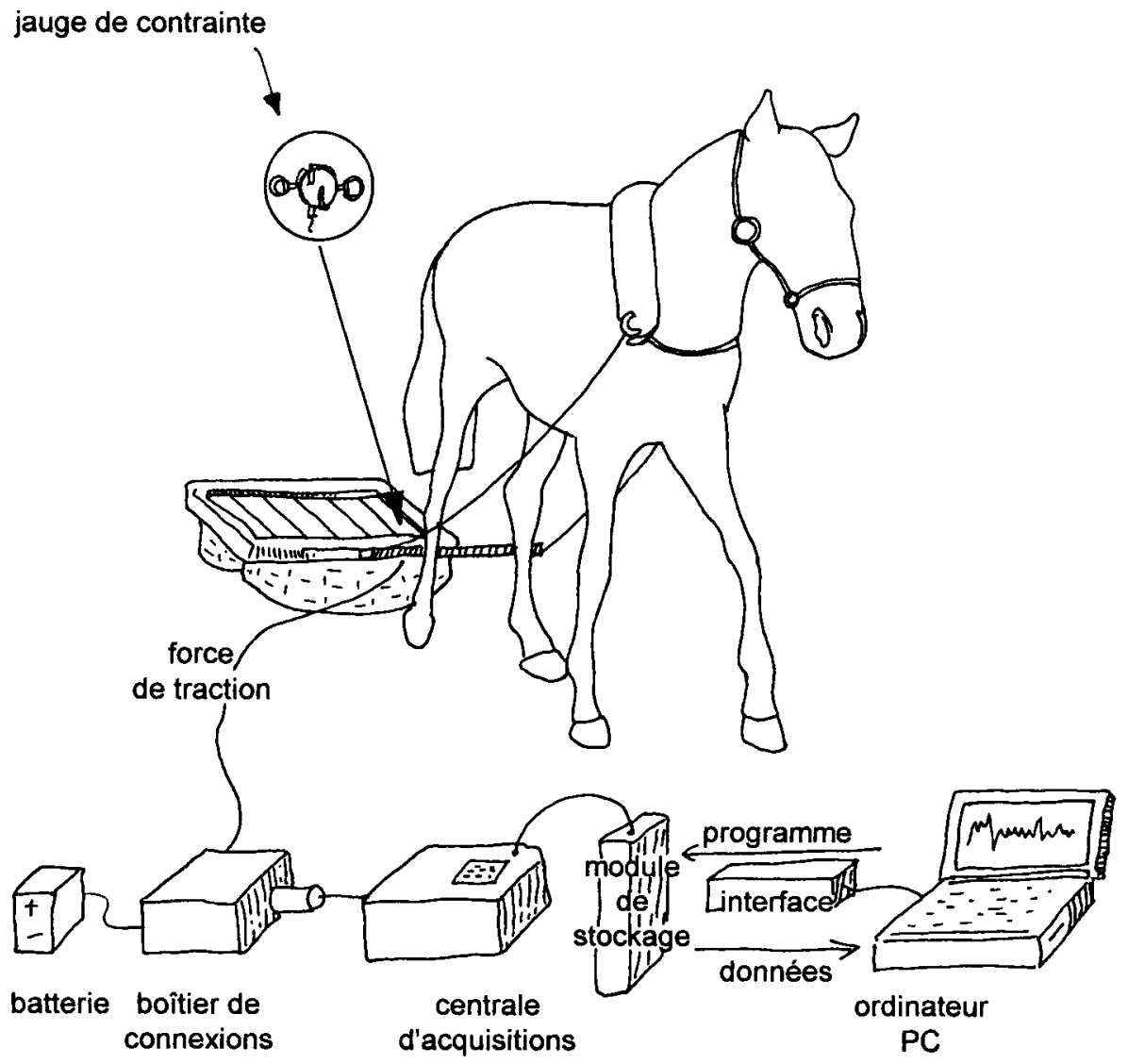

Figure 1. Chaîne de mesures Cirad : positionnement des capteurs.

\subsection{Séance d'essai}

Au cours d'une séance d'essai, un animal de poids vif connu (PV) tire seul, autour de la piste, un traîneau lesté de manière à atteindre une force de traction définie au préalable dans une gamme étalon. Cette force est maintenue constante tout au long de la séance (c'est-àdire pour une durée allant de $30 \mathrm{~min}$ à $7 \mathrm{~h}$ selon la charge). Les opérateurs effectuent une saisie complète des paramètres à chaque tour de piste. Le départ est donné de la base de contrôle vers 7.00 le matin. À la fin de chaque tour, l'animal se repose durant 3 à 4 min. En saison pluvieuse, la séance d'essai est interrompue lorsque l'animal manifeste des signes de fatigue évidents (ralentissement, arrêts, couchers, charges, etc.) ; en saison sèche chaude, la séance est interrompue à 11.00 en raison des fortes chaleurs de la mi-journée.

Les harnachements sont un collier pour le cheval, une bricole pour l'âne et un jouguet de type chinois pour le bovin. Les deux modèles de traîneaux sont constitués d'un plateau en bois boulonné sur deux pneus (petit modèle : poids total à vide $19 \mathrm{~kg}$, pneus $185 \times 15$; grand modèle : poids total à vide $53 \mathrm{~kg}$, pneus $900 \times$ 20). 
Le jour de la séance d'essai, l'animal choisi est frais ( $3 \mathrm{j}$ de repos entre deux essais) et entraîné ( 2 à 3 entraînements de 2 h chaque semaine). Il est préparé à 6.00 le matin (distribution du concentré alimentaire et abreuvement ; marche sur $1 \mathrm{~km}$ de l'écurie à la base de contrôle ; appareillage ; lestage du traîneau). Au terme de la séance d'essai, l'animal est mis au repos durant trois jours, ne sortant que pour se rendre au pâturage.

Les séances d'essais sont effectuées en saison des pluies (juin à septembre) et en saison sèche chaude (mars à avril). La saison des pluies correspond à la période des cultures. Elle se caractérise par une forte hygrométrie et une température moyenne pour la région (tableau II). La saison sèche chaude se caractérise par une température très élevée et une hygrométrie moyenne.

Quatre paliers de force de traction sont choisis : $8,10,15$ et $20 \% \mathrm{PV}$ pour les bovins ; 10 , 15,20 et $25 \% \mathrm{PV}$ pour les équidés. Au total, 133 séances d'essai équitablement réparties entre les espèces et les saisons ont été réalisées.

Le tableau III présente l'enregistrement d'une séance d'essai. Dans le texte, la valeur mesurée d'une variable « $y$ » est notée $\mathrm{Y}_{\mathrm{m}}$ et sa valeur calculée par régression est notée $Y$.

Tableau II. Températures et hygrométries mesurées à Garoua en 1992 en saison sèche chaude et en saison pluvieuse à différentes heures de la journée encadrant les séances d'essais (source : Ira Garoua).

\begin{tabular}{lccc}
\hline & & Heures & \\
\cline { 2 - 4 } Saisons & 7.00 & 11.00 & 13.00 \\
\hline $\begin{array}{l}\text { Saison sèche chaude } \\
\text { (mars et avril) }\end{array}$ & $28^{\circ} \mathrm{C} ; 54 \%$ & $35^{\circ} \mathrm{C} ; 37 \%$ & $37^{\circ} \mathrm{C} ; 30 \%$ \\
$\begin{array}{l}\text { Saison pluvieuse } \\
\text { juin à septembre) }\end{array}$ & $25{ }^{\circ} \mathrm{C} ; 100 \%$ & $27^{\circ} \mathrm{C} ; 89 \%$ & $28{ }^{\circ} \mathrm{C} ; 84 \%$ \\
\hline
\end{tabular}

Tableau III. Séance d'essai du cheval Mustang effectuée le 8 mars 1994.

\begin{tabular}{lcccccc}
\hline Tours piste & $\begin{array}{c}\mathrm{L}_{\text {cum }} \\
(\mathrm{m})\end{array}$ & $\begin{array}{c}\mathrm{d}_{\text {tour }} \\
(\mathrm{min})\end{array}$ & $\begin{array}{c}\mathrm{d}_{\text {cum }} \\
(\mathrm{min})\end{array}$ & $\begin{array}{c}\mathrm{PV} \\
(\mathrm{kg})\end{array}$ & $\begin{array}{c}\mathrm{F}_{\mathrm{i}} \\
(\mathrm{kg})\end{array}$ & $\begin{array}{c}\mathrm{v}_{\mathrm{i}} \\
(\mathrm{m} / \mathrm{s})\end{array}$ \\
\hline $1^{\mathrm{er}}$ tour & 1245 & 18 & 18 & 280 & 36,99 & 1,15 \\
$2^{\mathrm{e}}$ tour & 2490 & 20 & 38 & 280 & 37,85 & 1,04 \\
$3^{\mathrm{e}}$ tour & 3735 & 20 & 58 & 280 & 41,66 & 1,04 \\
$4^{\mathrm{e}}$ tour & 4980 & 25 & 83 & 280 & 42,75 & 0,83 \\
$5^{\mathrm{e}}$ tour & 6225 & 21 & 104 & 280 & 43,65 & 0,99 \\
$6^{\mathrm{e}}$ tour & 7470 & 24 & 128 & 280 & 39,13 & 0,86 \\
$7^{\mathrm{e}}$ tour & 8715 & 25 & 153 & 280 & 40,00 & 0,83 \\
$8^{\mathrm{e}}$ tour & 9960 & 25 & 178 & 280 & 40,00 & 0,83 \\
$+300 \mathrm{~m}$ & 10260 & 7 & 185 & 280 & 40,00 & 0,71 \\
& & & & & & \\
Moyennes & $\mathrm{L}_{\mathrm{m}}$ & & $\mathrm{d}_{\mathrm{m}}$ & $\mathrm{PV}$ & $\mathrm{F}$ & $\mathrm{v}_{\mathrm{m}}$ \\
(Y & 10260 & & 185 & 280 & 40,50 & 1,08
\end{tabular}

Palier de force de traction fixé à $15 \% P V . L_{\text {cum }}$ : distance parcourue cumulée $; d_{\text {tour }}:$ durée du tour ; $d_{\text {cum }}:$ durée cumulée $; P V:$ poids vif $; F_{i}$ : force de traction au tour $i ; v_{i}:$ vitesse au tour $i\left(=1245 / \mathrm{d}_{\text {tour }}\right) ; L_{m}:$ distance parcourue $; \mathrm{d}_{\mathrm{m}}$ : durée totale $; \mathbf{F}$ : force de traction moyenne $; \mathrm{v}_{\mathrm{m}}:$ vitesse moyenne. 


\section{RÉSULTATS}

\subsection{Travail et force de traction}

\subsubsection{Distance parcourue et force de traction}

Pour chaque espèce, la distance parcourue $L_{m}$ (en $m$ ) est en relation linéaire avec la force de traction $F(e n \% P V)$ sur l'intervalle compris entre 8 et $25 \% \mathrm{PV}$ (figure 2) :

$$
\mathrm{L}=\mathrm{b}+\mathrm{a} \cdot \mathrm{F} \text {; }
$$

$$
\text { cheval : } \mathrm{L}=37091-1440 \cdot \mathrm{F}
$$

$$
\begin{aligned}
& \left(\mathrm{R}^{2}=0,91 ; \mathrm{ddl}=22 ; \mathrm{p} \leq 0,01\right) ;[2] \\
& \quad \text { zébu }: \mathrm{L}=30316-1247 \cdot \mathrm{F} \\
& \left(\mathrm{R}^{2}=0,92 ; \mathrm{ddl}=20 ; \mathrm{p} \leq 0,01\right) ;[3] \\
& \quad \text { âne }: \mathrm{L}=25329-976 \cdot \mathrm{F} \\
& \left(\mathrm{R}^{2}=0,92 ; \mathrm{ddl}=22 ; \mathrm{p} \leq 0,01\right) . \quad[4]
\end{aligned}
$$

Lorsque la force de traction dépasse $25 \% \mathrm{PV}$ (pour les équidés), $20 \% \mathrm{PV}$ (pour les bovins), les animaux refusent d'avancer. Pour une force de traction comprise

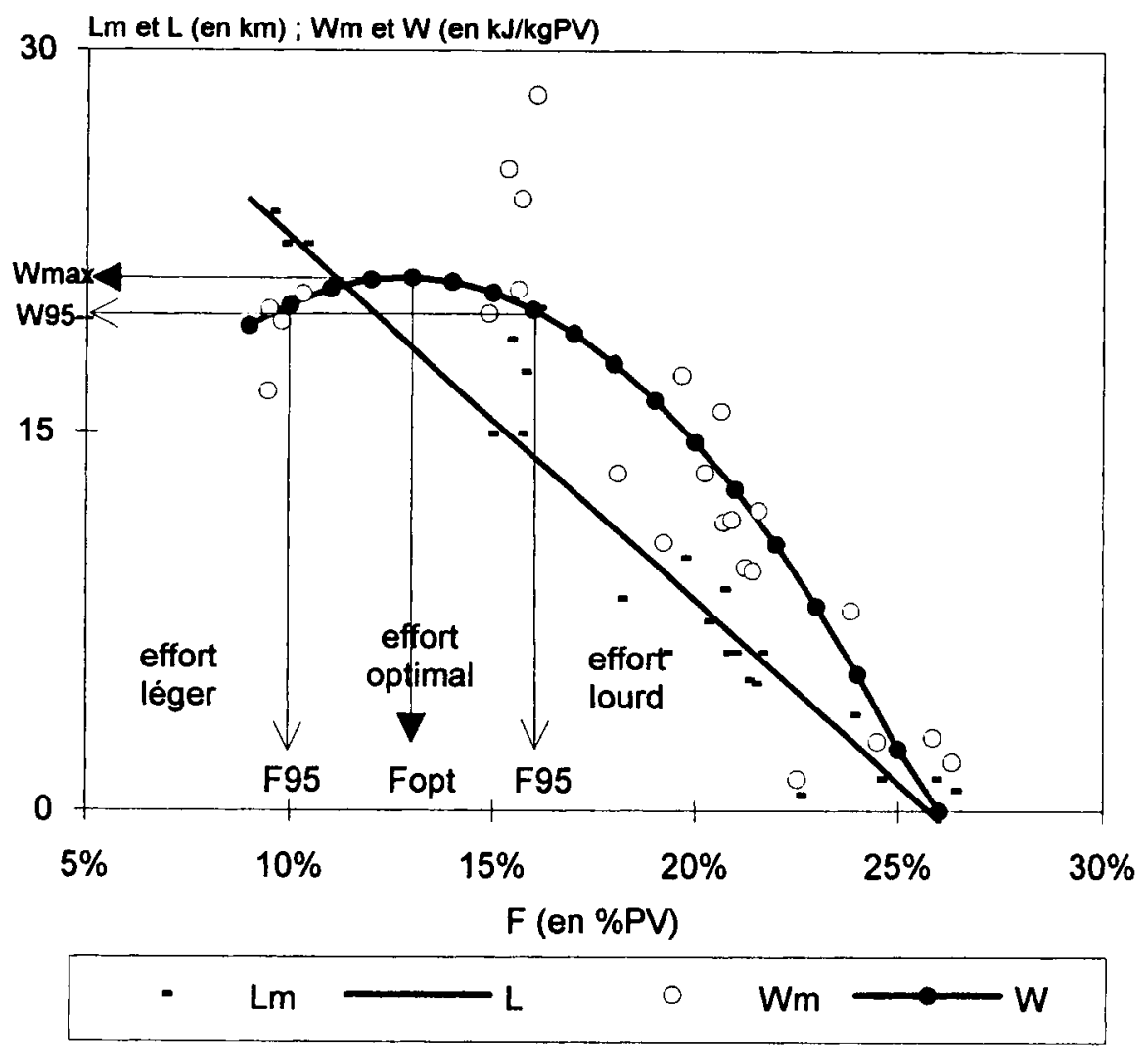

Figure 2. Distance parcourue (mesurée : $\mathrm{L}_{\mathrm{m}}$; et ajustée : L) et travail de traction (mesuré : $\mathrm{W}_{\mathrm{m}}$; et ajusté : W) en fonction de la force de traction $(\mathrm{F})$ : cas du cheval, PV : poids vif $(\mathrm{kg})$; $\mathrm{F}$ : force de traction $(\% \mathrm{PV}) ; \mathrm{L}(\mathrm{km})=(3709-1440 \cdot \mathrm{F}) / 1000 ; \mathrm{W}(\mathrm{kJ} / \mathrm{kg} \mathrm{PV})=9,81 . \mathrm{F} \cdot \mathrm{L} \cdot 0,9 /$ $100 ;$ Fopt $(\% \mathrm{PV})=3709 /(2 \cdot 1440) ; \mathrm{Wmax}(\mathrm{kJ} / \mathrm{kgPV})=9,81 \cdot$ Fopt $\cdot \mathrm{L}($ Fopt $) \cdot 0,9 / 100 ; \mathrm{W} 95=$

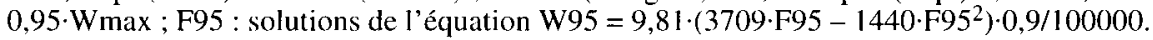


entre 0 et $5 \% \mathrm{PV}$, la distance parcourue est pratiquement proportionnelle à la durée de l'exercice, car l'animal ralentit très peu. Lors de séances de marche sans traction (durée $8 \mathrm{~h}$ ), les vitesses moyennes enregistrées sont pratiquement constantes du début à la fin : cheval : $1,00 \pm 0,1 \mathrm{~m} / \mathrm{s}$; âne : $0,90 \pm 0,1 \mathrm{~m} / \mathrm{s}$; zébu : $0,90 \pm 0,1$ $\mathrm{m} / \mathrm{s}$.

\subsubsection{Stabilité de la force de traction au cours du temps}

Le ralentissement de l'attelage induit une réduction de la force de frottement jouant sur l'interface sol-traîneau, ce qui entraîne une baisse de la force de traction. Pour quantifier ce phénomène, nous avons étudié l'évolution du rapport $\mathrm{F}_{\mathrm{i}} / \mathrm{F}_{0}\left(\mathrm{~F}_{\mathrm{i}}\right.$ : force de traction au tour $\mathrm{i} ; \mathrm{F}_{0}$ : force de traction au premier tour) en fonction de la vitesse de l'attelage $\left(\mathrm{v}_{\mathrm{i}}\right.$ : vitesse de l'attelage au tour i). Dans le cas du cheval, pour le palier $\mathrm{F}=15 \% \mathrm{PV}$, la régression n'est pas significative : $F_{i} / F_{0}=1,019+0,07 \cdot v_{i}$ $\left(R^{2}=0,0004 ; d d l=53\right)$. Pour les deux autres espèces et pour l'ensemble des traitements aucune corrélation significative (au seuil de $5 \%$ ) n'a été mise en évidence. La force de traction ne varie donc pas significativement avec la vitesse de marche.

\subsubsection{Travail et force de traction: plage d'efficacité maximale}

Un animal de poids vif PV (en $\mathrm{kg}$ ) qui effectue un effort de traction F (en \% PV) sur une distance $\mathrm{L}_{\mathrm{m}}$ (en $\mathrm{m}$ ), produit un travail de traction $\mathrm{W}_{\mathrm{m}}$ (en $\mathrm{kJ} / \mathrm{kgPV}$ ) dont l'expression est la suivante :

$$
\mathrm{W}_{\mathrm{m}}=9,81 \cdot \mathrm{F} \cdot \mathrm{L}_{\mathrm{m}} \cdot \cos \theta \cdot 10^{-5}
$$

( $\theta$ : angle de traction mesuré de $25^{\circ}$ environ, soit $\cos \theta=0,90$ ). Comme le suggèrent Betker et Kutzback [1], les équations [2], [3] et [4] portées dans l'équation [5] conduisent à l'expression suivante du travail de traction (W) :

$$
\begin{gathered}
W=9,81 \cdot\left(b \cdot F+a \cdot F^{2}\right) \cdot 0,9 \cdot 10^{-5} \\
\text { cheval }: W=9,81 \cdot \\
\left(37091 \cdot F-1440 \cdot F^{2}\right) \cdot 0,9 \cdot 10^{-5} \\
\text { zébu : } W=9,81 \\
\left(30316 \cdot F-1247 \cdot F^{2}\right) \cdot 0,9 \cdot 10^{-5} \\
\text { âne }: W=9,81 \cdot \\
\left(25329 \cdot F-976 \cdot F^{2}\right) \cdot 0,9 \cdot 10^{-5}
\end{gathered}
$$

La valeur du travail maximum (notée $\mathrm{W}_{\text {max }}$; figure 2) est donnée par la valeur de $\mathrm{F}$ qui annule la dérivée de l'équation [6], appelée pour cette raison force optimale de traction (notée $\mathrm{F}_{\text {opt }}$ ). À partir des équations [7], [8] et [9] $F_{\text {opt }}$ s'obtient comme suit :

$$
\begin{gathered}
\mathrm{dW} / \mathrm{dF}=\mathrm{b}+2 \cdot \mathrm{a} \cdot \mathrm{F} ; \\
\text { soit }: \mathrm{F}_{\text {opt }}=-\mathrm{b} /(2 \cdot \mathrm{a}) ; \\
\text { cheval : } \\
\mathrm{F}_{\mathrm{opt}}=37091 /(2 \cdot 1440)=12,88 \% \mathrm{PV} ; \\
\text { zébu : } \\
\mathrm{F}_{\text {opt }}=30316 /(2 \cdot 1247)=12,15 \% \mathrm{PV} ; \\
\text { âne : } \\
\mathrm{F}_{\text {opt }}=25329 /(2 \cdot 976)=12,98 \% \mathrm{PV} .
\end{gathered}
$$

Une analyse de variance effectuée sur les valeurs de $F_{\text {opt }}$ calculées pour chaque individu de l'essai (quatre répétitions par espèce) indique que les différences entre les $\mathrm{F}_{\mathrm{opt}}$ des trois espèces ne sont pas significatives $(p=0,08)$.

La plage d'efficacité maximale est définie arbitrairement comme l'intervalle, centré sur $\mathrm{F}_{\text {opt }}$ dans lequel l'animal accomplit un travail supérieur à $0,95 \cdot \mathrm{W}_{\max }$ (noté $\mathrm{W}_{95}$; figure 2 ). Les valeurs de $\mathrm{F}$ correspondantes (notées $\mathrm{F}_{95}$ bornes de $\mathrm{F}_{\text {opt }}$ $\pm \delta_{95}$ ) sont données par les solutions de l'équation suivante :

$$
\mathrm{W}_{95}=9,81 \cdot\left(\mathrm{b} \cdot \mathrm{F}+\mathrm{a} \cdot \mathrm{F}^{2}\right) \cdot 0,9 \cdot 10^{-5} \text {. }
$$

Pour les trois espèces, les bornes de la plage d'efficacité maximale sont :

$$
\begin{gathered}
\text { cheval : } \mathrm{F}_{\text {opt }}=(12,88 \pm 2,88) \% \mathrm{PV} \\
\text { soit } 10,0 \text { et } 15,8 \% \mathrm{PV} ; \\
\text { zébu : } \mathrm{F}_{\text {opt }}=(12,15 \pm 2,72) \% \mathrm{PV} \\
\text { soit } 9,43 \text { et } 14,9 \% \mathrm{PV} ;
\end{gathered}
$$


âne : $F_{\text {opt }}=(12,98 \pm 2,90) \% \mathrm{PV}$ soit 10,1 et $15,9 \% \mathrm{PV}$.

Les bornes de ces plages, bien que différentes en raison du mode de calcul, ne peuvent pas être considérées comme significativement différentes, compte tenu du résultat de l'analyse de variance effectuée plus haut sur $\mathrm{F}_{\text {opt }}$. Quelle que soit l'espèce, on retiendra finalement que, lorsque la force de traction est inférieure à $10 \% \mathrm{PV}$ (effort de traction « léger »), c'est la vitesse de l'animal qui limite la production de travail, alors que lorsque la force de traction dépasse $15 \%$ PV c'est l'intensité de l'effort qui limite la production de travail (effort de traction « lourd »).

\subsection{Durée et vitesse de travail}

\subsubsection{Durée moyenne du travail}

\subsubsection{Cas du cheval}

La durée moyenne de travail d'un cheval (notée $d_{m}$ en min) varie en fonction de la force de traction (figure 3). Pour des efforts «légers» (moins de $10 \% \mathrm{PV}$ ), l'arrêt de la séance est davantage commandé par la fatigue des conducteurs que par celle des animaux (au bout de $7 \mathrm{~h}$ ). En revanche, pour des efforts « lourds » (plus de $20 \%$ PV) l'arrêt de la séance d'essai dépend de la résistance des animaux qui manifestent des signes évidents de fatigue.

Compte tenu de la structure du nuage de points $\left(\mathrm{F} ; \mathrm{d}_{\mathrm{m}}\right)$, une régression a été établie à l'aide d'un ajustement quadratique (figure 3 ) : $\mathrm{d}=752,33-38,03 \cdot \mathrm{F}+$ $0,43 \cdot \mathrm{F}^{2}\left(\mathrm{R}^{2}=0,88 ; \mathrm{ddl}=23\right.$; avec $\mathrm{d}$ en min et $\mathrm{F}$ en \%PV). La part de la variabilité expliquée par le modèle est acceptable $\left(\mathrm{R}^{2}=0,88\right)$. Les résidus sont répartis de façon homogène autour de l'axe : $d_{m}-$ $\mathbf{d}=0$. Le test global du modèle conduit à une valeur du $F$ de Fischer $(85,85)$ élevée et très significative (modèle globalement bon). La composante quadratique est peu significative (conservée néanmoins car elle améliore la part de variabilité expliquée par le modèle).
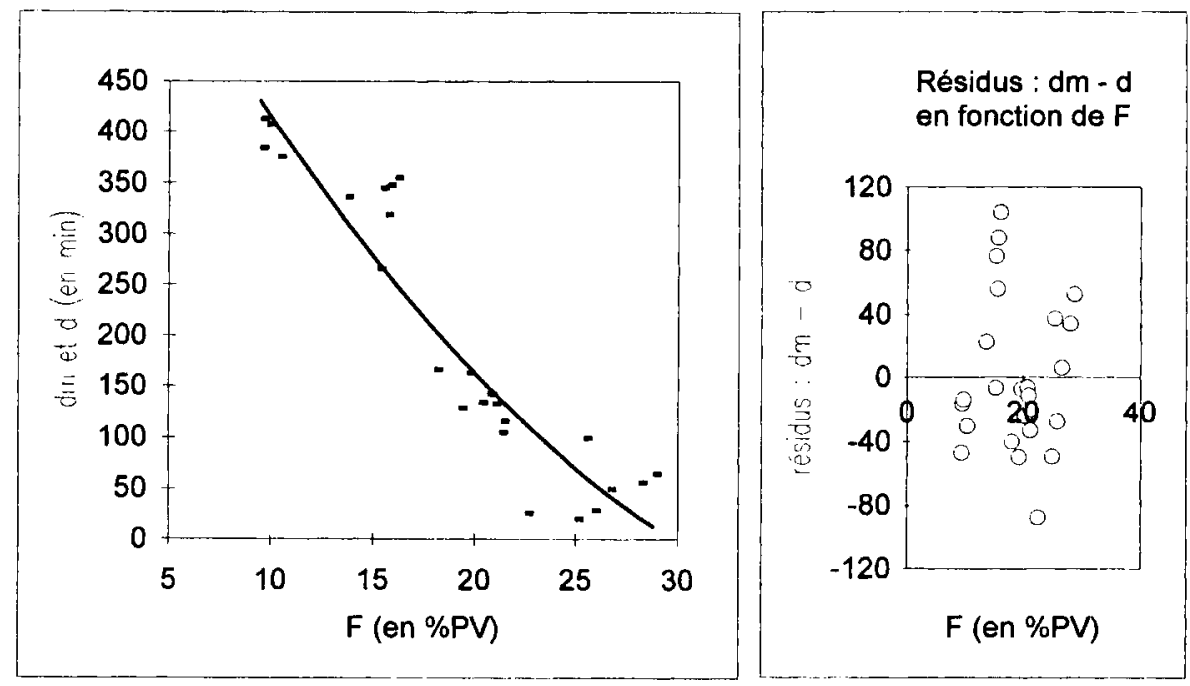

Figure 3. Variation de la durée du travail du cheval (mesurée $: d_{m} ;$ et ajustée : d) en fonction de la force de traction $(F)$. PV : poids vif. 


\subsubsection{Comparaison cheval, âne et zébu}

Des régressions analogues ont été établies chez le zébu et chez l'âne (figure 4). Pour une force de traction inférieure à $8 \% \mathrm{PV}$, la durée du travail a été limitée arbitrairement à $7 \mathrm{~h}$ (pour le zébu et le cheval) et à $6 \mathrm{~h}$ pour l'âne. Finalement, les durées moyennes de travail des trois espèces suivent un schéma similaire (tableau IV) : constantes pour $\mathrm{F}<$ à $8 \% \mathrm{PV}$ puis fortement décroissantes pour $\mathrm{F}$ comprise entre 8 et $25 \% \mathrm{PV}$. En procédant à une analyse de variance sur $\mathrm{d}$, on montre que pour $\mathrm{F}=10$ et $15 \% \mathrm{PV}$, les différences de $\mathrm{d}$ sont hautement significatives entre espèces ( $p<0,001)$, alors que pour $\mathrm{F}=20 \% \mathrm{PV}$, les différences sont peu significatives $(p<0,05)$.

\subsubsection{Vitesse moyenne}

\subsubsection{Cas du cheval}

La vitesse moyenne $\left(\mathrm{v}_{\mathrm{m}}\right)$ se calcule par le rapport : $\mathrm{L}_{\mathrm{m}} / \mathrm{d}_{\mathrm{m}}$. La structure du nuage de points $\left(\mathrm{F} ; \mathrm{v}_{\mathrm{m}}\right)$ nous a conduit à calculer une régression ajustée de façon qua- dratique (figure 5) : $\mathrm{v}=0,867-0,014 \cdot \mathrm{F}$ $+0,0008 \cdot \mathrm{F}^{2}\left(\mathrm{R}^{2}=0,29 ; \mathrm{ddl}=23\right.$; avec $\mathrm{v}$ en $\mathrm{m} / \mathrm{s}$ et $\mathrm{F}$ en $\% \mathrm{PV}$ ). La part de la variabilité expliquée par ce modèle est faible $(0,29)$ et les coefficients de degré un et deux sont peu significatifs. Le test global du modèle conduit à une valeur du $F$ de Fischer significative $(4,56$; modèle globalement bon). Il existe une variabilité importante de la vitesse moyenne entre les individus qui découle de leur différence de caractère. L'appréciation de la vitesse moyenne pourrait être améliorée par un plus grand nombre d'observations par espèce et par la prise en compte du facteur animal dans le modèle de v. Cet approfondissement serait indispensable, si l'étude portait sur une large gamme de vitesses (pas, trop, galop), or ce n'est pas le cas dans les conditions d'expérimentations présentées ici (marche entre 2,5 et $3,5 \mathrm{~km} / \mathrm{h}$ ).

\subsubsection{Comparaison cheval, ane et zébu}

Des régressions analogues ont été établies chez le zébu et chez l'âne (figure 6).

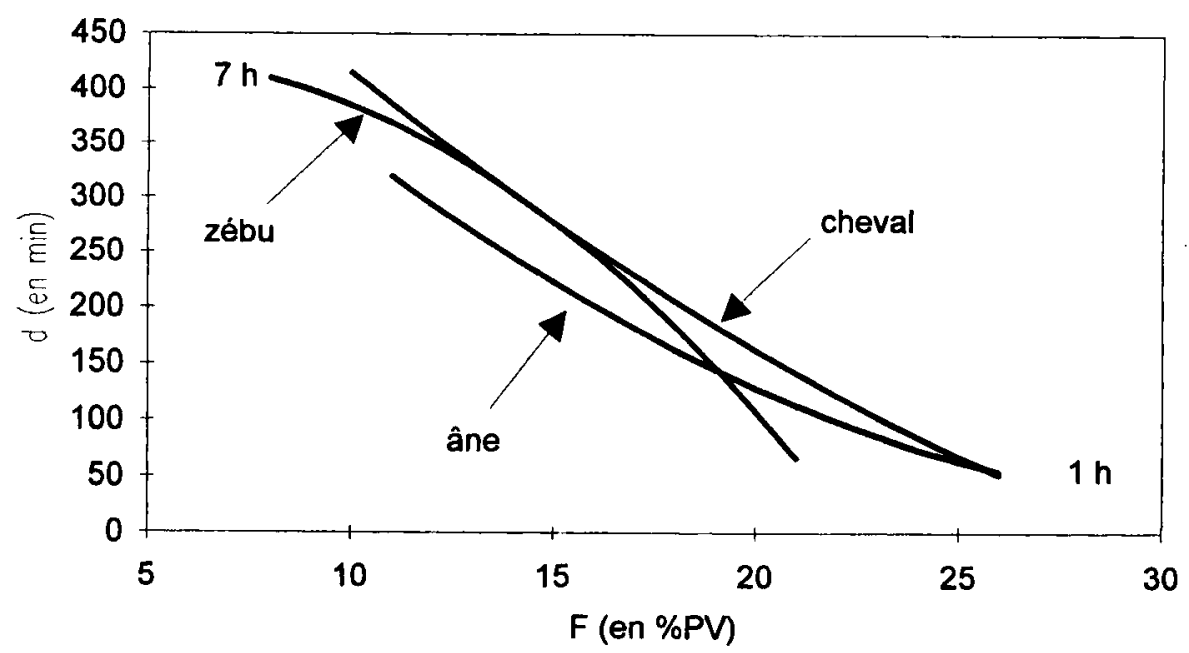

Figure 4. Variation de la durée moyenne du travail (d) en fonction de la force de traction $(\mathrm{F})$ : comparaison cheval, âne et zébu. PV : poids vif. 
Tableau IV. Durée moyenne de travail (d en min) selon la force de traction chez l'âne, le cheval et le zébu.

\begin{tabular}{lcc}
\hline Espèces & $\mathrm{F}<8 \% \mathrm{PV}$ & $\mathrm{F}$ comprise entre 8 et $25 \% \mathrm{PV}$ \\
\hline Âne & $\mathrm{d}=360$ & $\mathrm{~d}=681,07-39,33 \cdot \mathrm{F}+0,06 \cdot \mathrm{F}^{2}\left(\mathrm{R}^{2}=88 ; \mathrm{ddl}=20\right)$ \\
Cheval & $\mathrm{d}=420$ & $\mathrm{~d}=752,33-38,03 \cdot \mathrm{F}+0,43 \cdot \mathrm{F}^{2}\left(\mathrm{R}^{2}=0,88 ; \mathrm{ddl}=23\right)$ \\
Zébu & $\mathrm{d}=420$ & $\mathrm{~d}=410,51+9,92 \cdot \mathrm{F}-1,25 \cdot \mathrm{F}^{2}\left(\mathrm{R}^{2}=85 ; \mathrm{ddl}=21\right)$
\end{tabular}

$F$ : force de traction ; PV : poids vif.

Tableau V. Vitesse moyenne ( $\mathrm{v}$ en $\mathrm{m} / \mathrm{s}$ ) selon la force de traction chez l'âne, le cheval et le zébu.

\begin{tabular}{lcc}
\hline Espèces & $\mathrm{F}<8 \% \mathrm{PV}$ & $\mathrm{F}$ comprise entre 8 et $25 \% \mathrm{PV}$ \\
\hline Âne & $\mathrm{v}=0,9$ & $\mathrm{v}=1,28-0,05 \cdot \mathrm{F}+0,0008 \cdot \mathrm{F}^{2}\left(\mathrm{R}^{2}=62 ; \mathrm{ddl}=20\right)$ \\
Cheval & $\mathrm{v}=1,0$ & $\mathrm{v}=0,867+0,014 \cdot \mathrm{F}-0,0008 \cdot \mathrm{F}^{2}\left(\mathrm{R}^{2}=29 ; \mathrm{ddl}=23\right)$ \\
Zébu & $\mathrm{v}=0,9$ & $\mathrm{v}=1,09-0,04 \cdot \mathrm{F}+0,007 \cdot \mathrm{F}^{2}\left(\mathrm{R}^{2}=86 ; \mathrm{ddl}=21\right)$ \\
\hline
\end{tabular}

F : force de traction ; PV : poids vif.
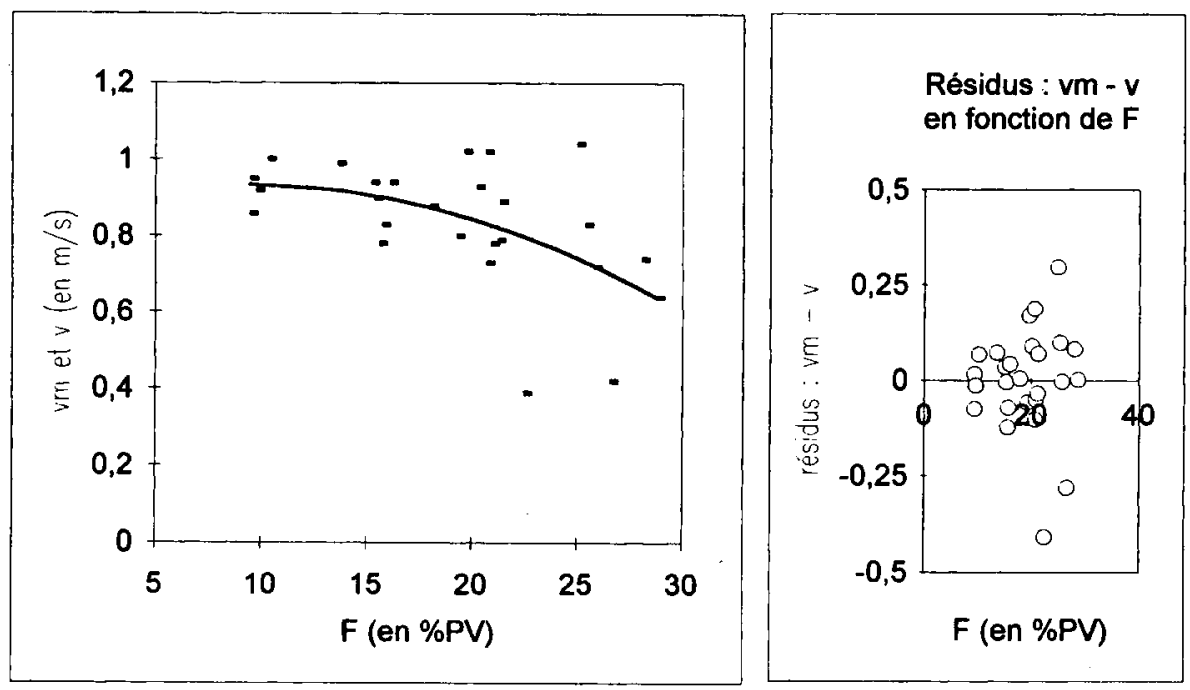

Figure 5. Variation de la vitesse moyenne du cheval (mesurée $: v_{m}$ et ajustée : v) en fonction de la force de traction $(\mathrm{F})$. $\mathrm{PV}$ : poids vif.

Pour $\mathrm{F}$ inférieure à $8 \% \mathrm{PV}$, nous avons estimé que la vitesse restait constante (on tend vers la marche sans traction). Finalement, les vitesses moyennes des trois espèces suivent un schéma similaire (tableau $\mathrm{V}$ ) : constantes pour $\mathrm{F}<$ à $8 \% \mathrm{PV}$ puis faiblement décroissantes pour $\mathrm{F}$ comprise entre 8 et $25 \% \mathrm{PV}$. En procédant à une analyse de variance sur $\mathrm{v}$, on montre que pour $\mathrm{F}=10 \% \mathrm{PV}$, les différences de $\mathrm{v}$ 


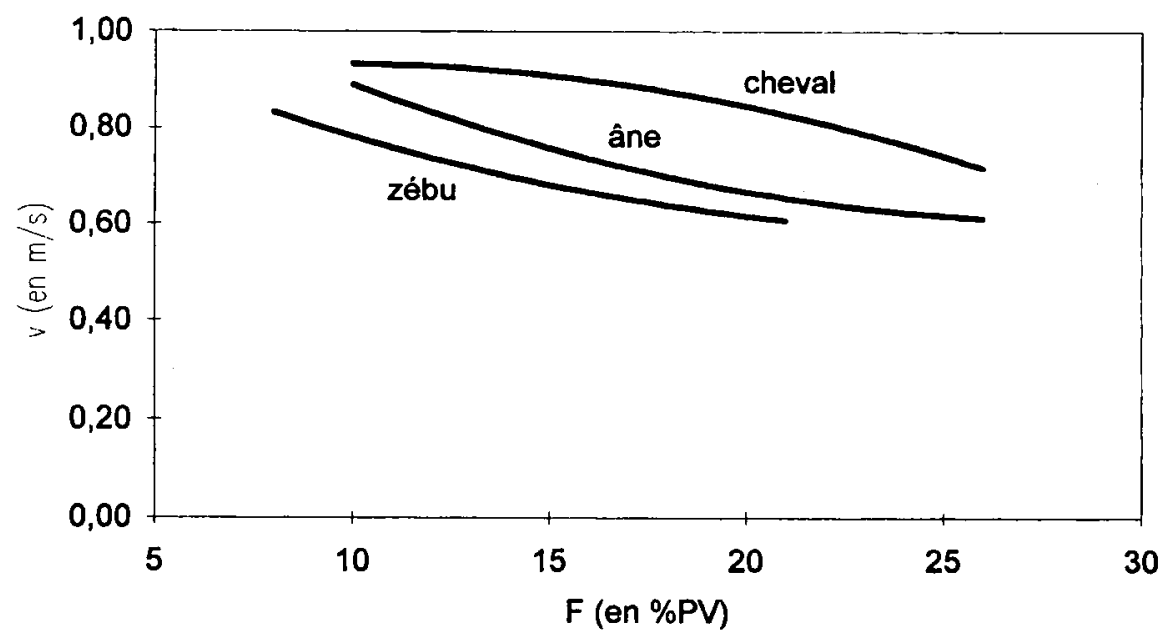

Figure 6. Variation de la vitesse moyenne de travail (v) en fonction de la force de traction (F) : comparaison cheval, âne et zébu. PV : poids vif.

entre espèces ne sont pas significatives $(p=0,025)$, pour $\mathrm{F}=15 \% \mathrm{PV}$, les différences sont significatives au seuil de $5 \%$ $(p=0,008)$, pour $\mathrm{F}=20 \% \mathrm{PV}$, les différences sont hautement significatives $(p<0,001)$.

\subsection{Dépense énergétique liée au travail}

La dépense énergétique liée au travail ( $\mathrm{DE}$ en $\mathrm{kJ}$ ) a été évaluée pour chaque espèce à l'aide d'une formule, établie par Lawrence et Stibbards [11], qui additionne les dépenses énergétiques élémentaires lors d'un effort de traction selon le modèle :

$\mathrm{DE}=\mathrm{DE}$ déplacement horizontal $+\mathrm{DE}$ travail de traction $+\mathrm{DE}$ portage $+\mathrm{DE}$ déplacement vertical

\subsubsection{Cas du cheval}

Le terme de la formule relatif au déplacement vertical a été négligé puisque la piste d'essai était pratiquement plate. Finalement, DE s'exprime en fonction de F et PV en effectuant deux opérations successives.

\subsubsection{Temps $I$}

Connaissant les expressions de la distance parcourue ( $\mathrm{L}$ en $\mathrm{km}$ ), du travail de traction ( $\mathrm{W}$ en $\mathrm{kJ}$ ) et de la charge portée ${ }^{3}$ (C en $\mathrm{kg}$ ) :

$$
\begin{gathered}
\mathrm{L}=(37091-1440 \cdot \mathrm{F}) \cdot 10^{-3} \\
\mathrm{~W}=9,81 \cdot \mathrm{F} \cdot \mathrm{PV} \\
(37091-1440 \cdot \mathrm{F}) \cdot \cos \theta \cdot 10^{-5} \\
\mathrm{C}=\mathrm{F} \cdot \mathrm{PV} \cdot \sin \theta \cdot 10^{-2}
\end{gathered}
$$

avec $\mathrm{F}$ en $\% \mathrm{PV}$; PV en $\mathrm{kg} ; \theta=25^{\circ}$.

\subsubsection{Temps 2}

On porte les équations (2), (7) et (11) dans la formule de Lawrence et Stibbards [11] $; \mathrm{DE}=\mathrm{a} \cdot \mathrm{L} \cdot \mathrm{PV}+\mathrm{W} / \mathrm{c}+\mathrm{b} \cdot \mathrm{L} \cdot \mathrm{C}$; en

${ }^{3} \mathrm{C}$ correspond à la composante verticale de la force de traction. 
Tableau VI. Coefficients ( $a, b$ et c) de la formule de la dépense énergétique liée au travail (DE).

\begin{tabular}{lccc}
\hline & \multicolumn{3}{c}{ Espèces } \\
\cline { 2 - 4 } Coefficients & cheval (1) & zébu (1) & âne (2) \\
\hline a : DE en kJ pour un déplacement de $1 \mathrm{~kg}$ de PV sur I m & 1,1 & 2,1 & 0,97 \\
b : DE en kJ pour le portage de I kg sur 1 m parcouru à plat & 1,1 & 2,6 & $1,1(3)$ \\
c: rendement absolu du travail de traction & 0,36 & 0,30 & 0,37 \\
\hline
\end{tabular}

PV : poids vif $(\mathrm{kg})$; $\mathrm{L}$ : distance parcourue $(\mathrm{en} \mathrm{km}) ; \mathrm{W}:$ travail de traction (en $\mathrm{kJ}) ; \mathrm{C}$ : composante verticale de la force de traction (en $\mathrm{kg}$ ); $\mathrm{DE}=\mathrm{a} \cdot \mathrm{L} \cdot \mathrm{PV}+\mathrm{W} / \mathrm{c}+\mathrm{b} \cdot \mathrm{L} \cdot \mathrm{C} ;$ (1) Lawrence et Stibbards [11] ; (2) Dijkman [3] ; (3) identique au cheval (choix de l'auteur).

choisissant les valeurs des paramètres ( $\mathrm{a}$, b et c) indiquées dans le tableau VI, on peut calculer DE (en $\mathrm{kJ})$ :

$$
\begin{gathered}
\mathrm{DE}=(37,091-1,440 \cdot \mathrm{F}) \\
\left(1,1+9,81 \cdot \mathrm{F} \cdot 0,9 \cdot 10^{-2} / 0,36\right. \\
\left.+1,1 \cdot \mathrm{F} \cdot 0,42 \cdot 10^{-2}\right) \cdot \mathrm{PV}
\end{gathered}
$$

\subsubsection{Cas du zébu et de l'âne}

Pour le zébu et l'âne, une démarche analogue conduit aux expressions suivantes de $\mathrm{DE}$ (en $\mathrm{kJ})$ :

$$
\begin{gathered}
\text { Zébu : } \mathrm{DE}=(30,316-1,247 \cdot \mathrm{F}) \\
\left(2,1+9,81 \cdot \mathrm{F} \cdot 0,9 \cdot 10^{-2} / 0,30\right. \\
\left.+2,6 \cdot \mathrm{F} \cdot 0,42 \cdot 10^{-2}\right) \cdot \mathrm{PV} \\
\text { Âne : } \mathrm{DE}=(25,329-0,976 \cdot \mathrm{F}) \\
\left(0,97+9,81 \cdot \mathrm{F} \cdot 0,9 \cdot 10^{-2} / 0,37\right. \\
\left.+1,1 \cdot \mathrm{F} \cdot 0,42 \cdot 10^{-2}\right) \cdot \mathrm{PV}
\end{gathered}
$$

\subsubsection{Application numérique}

La DE exprimée en $\mathrm{kJ}$ par kilogramme de poids métabolique $\left(\mathrm{kJ} \mathrm{PV}^{0,75}\right)$ pour chaque espèce facilite la comparaison.

\subsubsection{Pour une distance fixée}

La DE augmente proportionnellement à l'intensité de la force de traction.

\subsubsection{2. À l'échelle d'une séance d'essai (figure 7)}

Lorsque la force de traction est inférieure à $\mathrm{F}_{\text {opt }}$ (figure 2), la DE est élevée parce que l'animal se déplace sur une longue distance mais tire peu. Lorsque la force de traction est supérieure à $\mathrm{F}_{\text {opt, }}$ la DE chute rapidement car l'animal reduit fortement la distance parcourue. La dépense énergétique liée au travail (DE) au niveau des plages d'efficacité maximale est comprise entre : 373 et $316 \mathrm{~kJ} \mathrm{PV}^{0,75}$ chez le zébu, 325 et $298 \mathrm{~kJ} \mathrm{PV}^{0,75}$ chez le cheval et 167 et $156 \mathrm{~kJ} \mathrm{PV}^{0,75}$ chez l'âne.

\subsection{Table des performances}

La représentation des performances au travail des trois espèces dans une abaque commune (tableau VII) indique en fonction de deux facteurs simples à mesurer sur l'attelage, le poids vif (PV) qui caractérise la capacité de travail et la force de traction $(\mathrm{F})$ qui caractérise le travail de traction :

- d'une part, de manière qualitative, le type travail accompli (léger, optimal, lourd), autrement dit la plage d'efficacité maximale (intervalle $\mathrm{F}_{\mathrm{opt}} \pm \delta_{95}$ );

- d'autre part, de manière quantitative, la durée du travail (d), la vitesse moyenne de l'animal ( $v$ ) et sa dépense énergétique liée au travail (DE).

Chaque ligne du tableau VII est relative à un animal de poids vif donné (PV) et chaque colonne à une force de traction d'intensité donnée $(F)$. L'intervalle des 


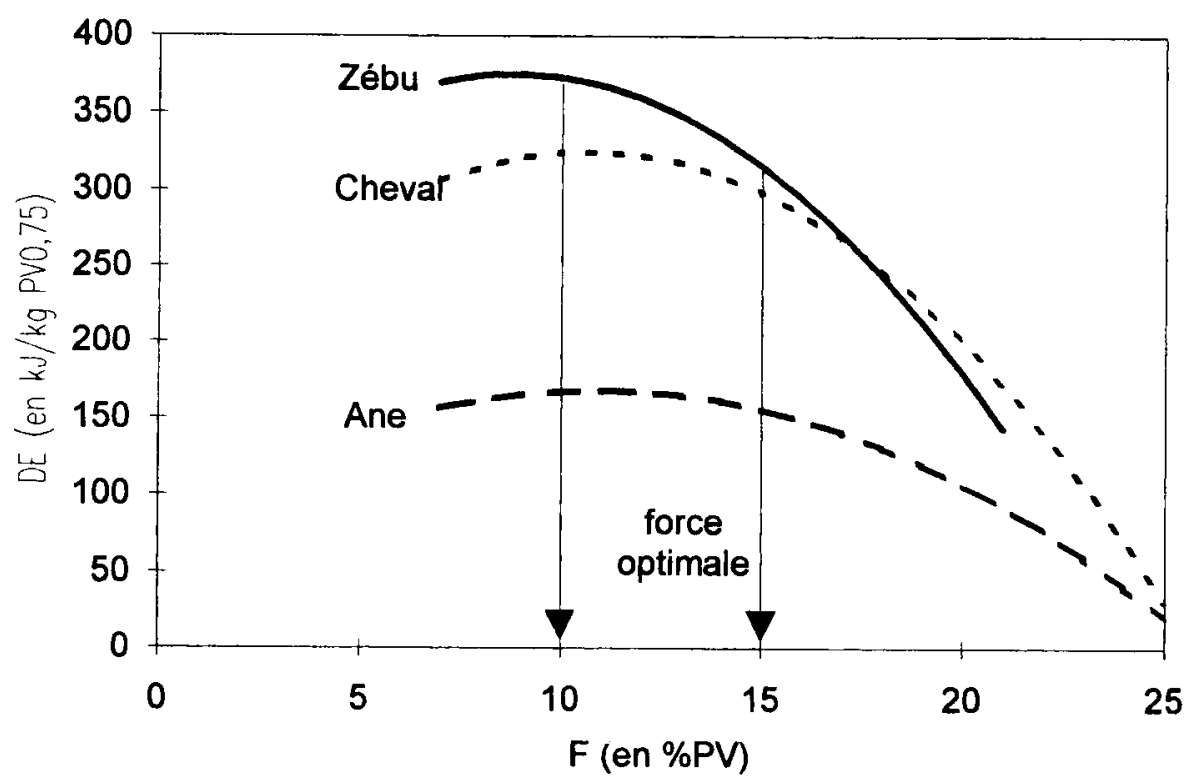

Figure 7. Variation de la dépense énergétique liée au travail (DE) en fonction de la force de traction $(F)$ chez le cheval, le zébu et l'âne : DE exprimée pour une séance d'essai.

poids vif (PV) retenus est 100 à $450 \mathrm{~kg}$ : 100 à $150 \mathrm{~kg}$ pour les ânes, 225 à $300 \mathrm{~kg}$ pour les chevaux et 325 à $450 \mathrm{~kg}$ pour les zébus. Les forces de traction s'échelonnent de 10 à $95 \mathrm{~kg}$, avec un incrément de $5 \mathrm{~kg}$ entre deux colonnes. À la croisée d'une ligne et d'une colonne figurent : la durée du travail ( $\mathrm{d}$ en $\mathbf{h}: \min$ ); la vitesse moyenne ( $v$ en $\mathrm{km} / \mathrm{h}$ ) ; la dépense énergétique liée au travail (DE en MJ). Les plages d'efficacité maximale sont en caractères gras. Les expression de $\mathrm{d}$, $\mathrm{v}$ et $D E$ définies plus haut en fonction de $F$ et de PV permettent de dresser un référentiel complet.

\section{DISCUSSION ET CONCLUSION}

\subsection{Commentaire des résultats}

Cette recherche précise les performances au travail des trois espèces ani- males de trait du Nord-Cameroun en fonction de deux facteurs : la force de traction (F) et le poids vif de l'animal (PV). Dans les conditions de l'essai, les autres facteurs susceptibles d'influencer l'efficacité du travail sont soit contrôlés au mieux, de façon à ce que leur effet reste le plus constant possible (entraînement, alimentation, santé etc.), soit équilibrés quand ils ne sont pas contrôlables (choix de plusieurs individus de tempérament différents, collecte des données égales entre les saisons, etc.).

Pour chaque espèce animale, une valeur optimale de la force de traction $\left(\mathrm{F}_{\text {opt }}\right)$ a été calculée. Par définition, un animal travaillant à $\mathrm{F}_{\text {opt }}$ produit un travail de traction maximal $\left(\mathrm{W}_{\max }\right)$. Les $\mathrm{F}_{\text {opt }}$ calculées (en \% PV) sont respectivement pour l'âne, le cheval et le zébu : 13,0 ; 13,0 ; et 12,0. La plage d'efficacité maximale est arbitrairement définie comme l'intervalle des 


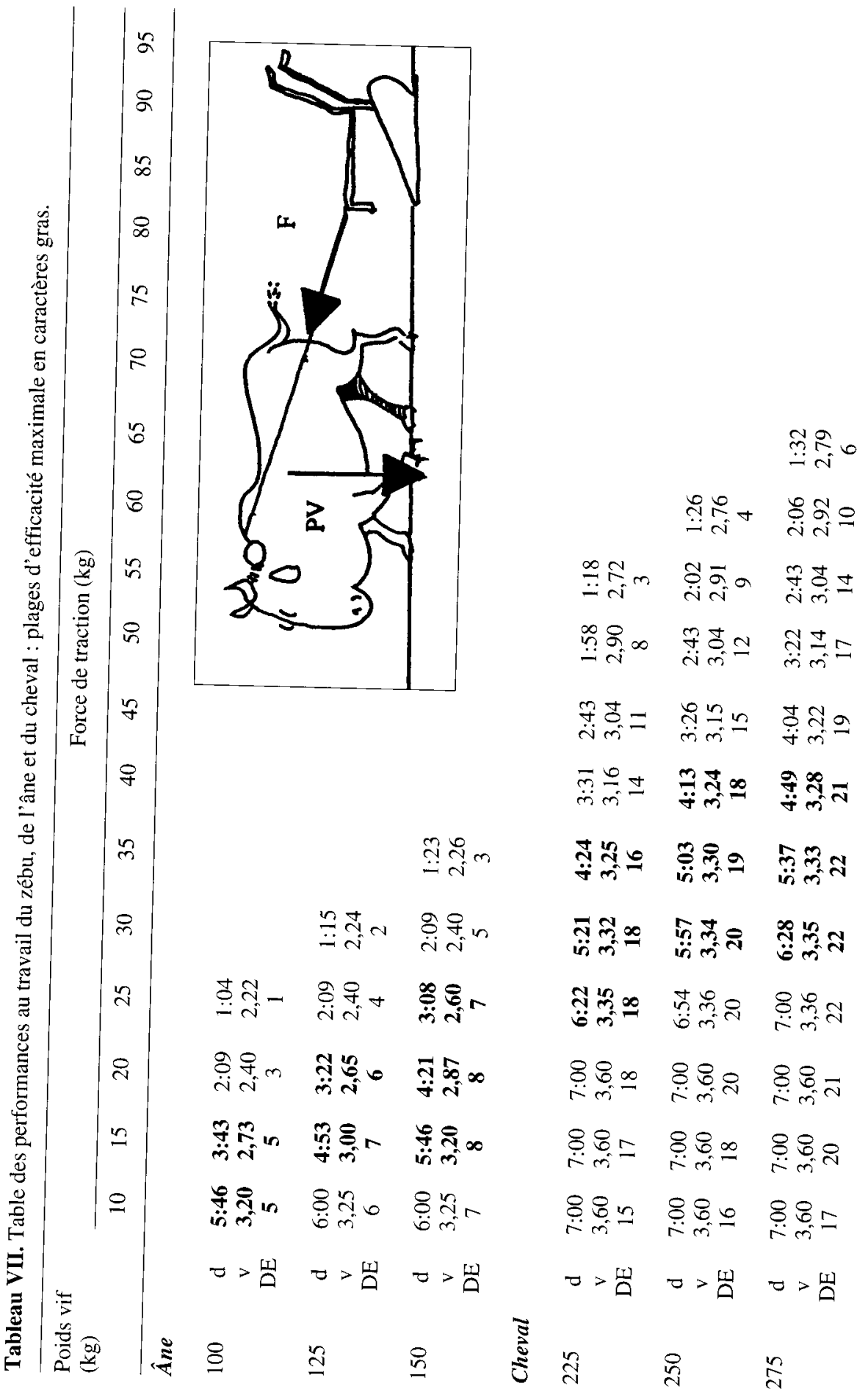


Capacités de travail du zébu, de l'âne et du cheval

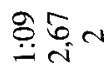

\begin{tabular}{|c|c|c|}
\hline & & 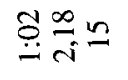 \\
\hline & $\stackrel{8}{\stackrel{8}{-} \stackrel{\infty}{4} \pm}$ & $\stackrel{\infty}{+} \underset{\sim}{\mathbb{i}} \Omega$ \\
\hline$\stackrel{\infty}{\infty} \frac{\infty}{i}=$ & $\stackrel{\infty}{\stackrel{i}{*} \underset{\sim}{N} \sim}$ & 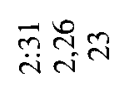 \\
\hline 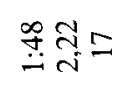 & শેّن & $\overline{\vec{m}} \overline{\vec{r}} \bar{N}$ \\
\hline
\end{tabular}

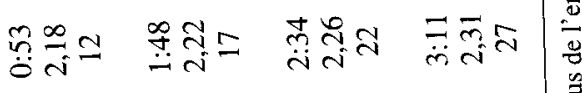

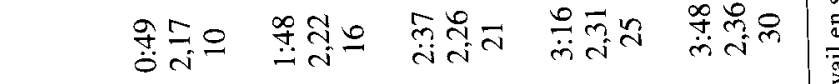

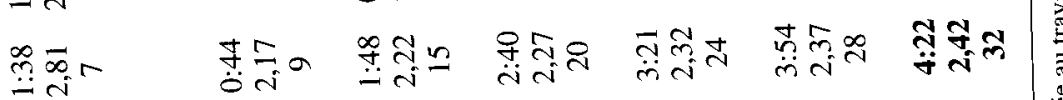

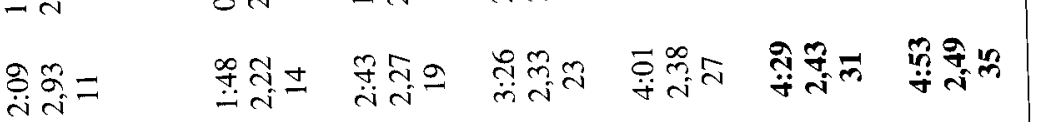

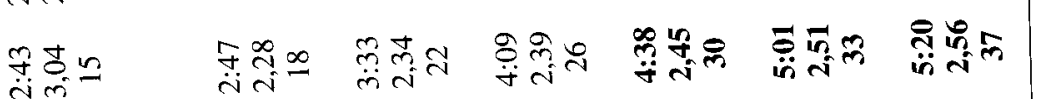

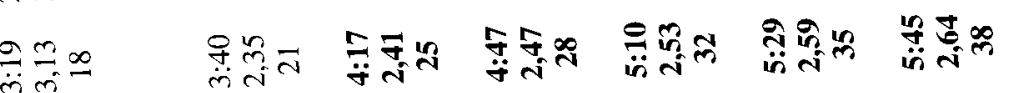

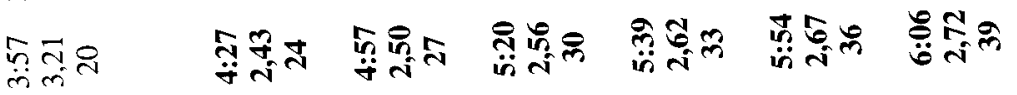

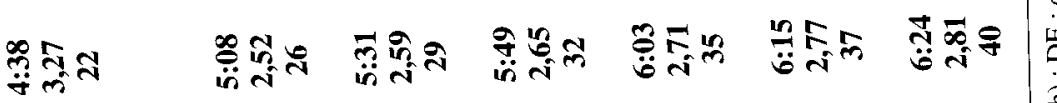

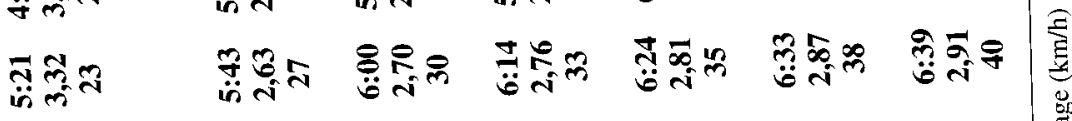

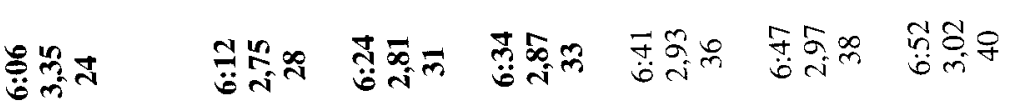

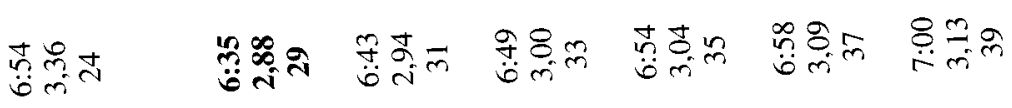

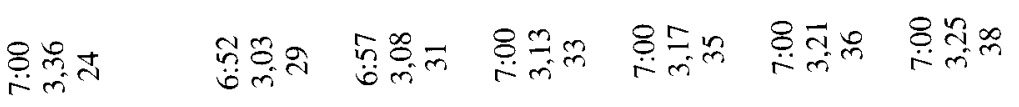

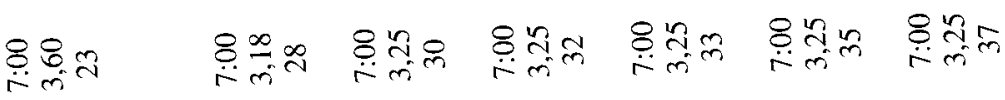

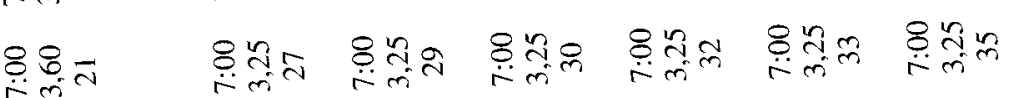

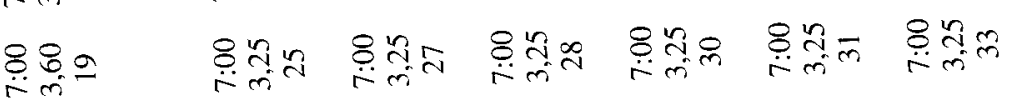

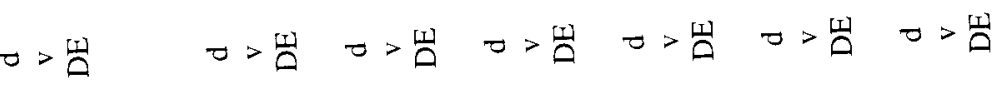

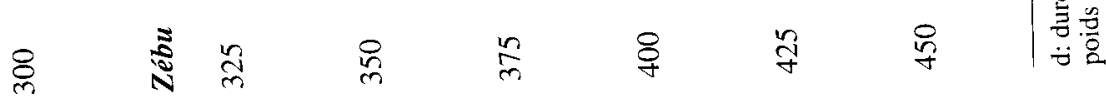


forces de traction centré sur $\mathrm{F}_{\text {opt }}$ à l'intérieur duquel le travail produit est supérieur à $0,95 \cdot \mathrm{W}_{\max }\left(\mathrm{F}_{\mathrm{opt}} \pm \delta_{95}\right)$. En dessous de cet intervalle, l'effort de traction est dit "léger» et au dessus, il est qualifié de « lourd $»$. Pour les trois espèces étudiées, les plages d'efficacité maximale calculées sont respectivement : $\mathrm{F}=10$ à $16 \% \mathrm{PV}$ pour les équidés; et $\mathrm{F}=9$ à $15 \% \mathrm{PV}$ pour les bovins. Aux bornes de ces plages, les durées moyennes de travail (d) et les vitesses moyennes $(\mathrm{v})$ sont les suivantes :

$$
\begin{gathered}
- \text { âne }: F=10 \% P V: d=5,5 h, \\
v=3,2 \mathrm{~km} / \mathrm{h} ; \mathrm{F}=16 \% \mathrm{PV}: \mathrm{d}=3,5 \mathrm{~h}, \\
\mathrm{v}=2,7 \mathrm{~km} / \mathrm{h} ; \\
- \text { cheval }: \mathrm{F}=10 \% \mathrm{PV}: \mathrm{d}=6,0 \mathrm{~h}, \\
\mathrm{v}=3,4 \mathrm{~km} / \mathrm{h} ; \mathrm{F}=16 \% \mathrm{PV}: \mathrm{d}=4,5 \mathrm{~h}, \\
\mathrm{v}=3,3 \mathrm{~km} / \mathrm{h} ; \\
- \text { zébu }: \mathrm{F}=9 \% \mathrm{PV}: \mathrm{d}=6,5 \mathrm{~h}, \\
\mathrm{v}=2,8 \mathrm{~km} / \mathrm{h} ; \mathrm{F}=15 \% \mathrm{PV}: \mathrm{d}=4,5 \mathrm{~h}, \\
\mathrm{v}=2,5 \mathrm{~km} / \mathrm{h} .
\end{gathered}
$$

Ces valeurs sont en bon accord avec diverses expériences africaines $[1,6,7$, 13]. Lorsque la force de traction augmente (de 10 à $20 \%$ PV), on constate des évolutions opposées du comportement des espèces en termes de durée et de vitesse de travail. Pour un effort léger $(10 \% \mathrm{PV})$, la durée de travail est significativement différente entre les espèces, alors que la vitesse ne l'est pas. Ce résultat indique une résistance à l'effort, entre espèces, différente : élevée chez le bovin, moyenne chez le cheval, plus faible chez l'âne. Pour un effort important ( $20 \% \mathrm{PV})$, la vitesse de travail est significativement différente entre espèces contrairement à la durée. Ce résultat traduit une différence de comportement sous la contrainte d'un effort violent : coup de collier du cheval, ralentissements élevés de l'âne et du bovin. Quelle que soit la force de traction, la vitesse moyenne du cheval est plus élevée que celles de l'âne et du zébu.

Pour un effort moyen, la dépense énergétique liée au travail résulte principalement du travail de traction, et secondai- rement du déplacement de l'animal. Pour un effort de faible intensité, l'énergie dépensée pour le déplacement peut dépasser celle de traction. Dans les conditions de l'essai, la diminution de la dépense énergétique liée au travail, lorsque la force de traction augmente, s'explique par la réduction des dépenses énergétiques de l'animal consenties pour sa locomotion. La formule de Lawrence et Stibbards [11], évalue la dépense énergétique liée au travail. On ne sait pas effectuer le passage de la dépense au besoin énergétique de l'animal dans le cas du travail. Les possibilités d'applications d'un tel résultat pour le développement sont donc limitées car la connaissance du besoin est primordiale pour raisonner le rationnement et le stockage des aliments.

\subsection{Critique de la méthode}

Les résultats présentés se réfèrent à un standard animal éloigné de la réalité des conditions paysannes puisqu'il s'agit d'animaux reposés, entraînés, convenablement nourris et soignés en milieu contrôlé. La table des performances à l'effort, bien que se référant à un travail de traction simplifié, met en relation les performances de trois espèces fréquemment attelées en Afrique subsaharienne, sur la base d'observations effectuées en milieu réel avec des animaux placés dans des conditions identiques. Le référentiel proposé mérite cependant d'être amélioré par des travaux en milieu paysan qui permettraient d'intégrer des facteurs de variation du travail, autres que F et PV, comme la gestion de l'effort sur la durée, l'état corporel et d'entraînement des animaux, etc.

Des contradictions sont apparues entre les simulations d'effort de traction sur piste et des travaux effectués en plein champ lorsque la force de traction dépasse $20 \% \mathrm{PV}$. Ceci ne remet pas en cause le 
choix initial d'une expérimentation en milieu contrôlé, car cette condition était indispensable pour obtenir des situations répétables et comparables entre individus et entre espèces avec des paliers fixés de force de traction. En plein champ, la présence de gradients (pente) ou de discontinuités (rupture de pente, différences pédologiques, etc.) modifient le comportement à l'effort. La table des performances se réfère à des conditions particulières de travail (rythme imposé par le tour de piste, effort de traction quasi constant) ce qui correspond en pratique à un travail sur terrain plat en sol humide. Des observations complémentaires ont montré que la validité de la table reste acceptable si la force de traction $(\mathrm{F})$ est inférieure à $20 \% \mathrm{PV}$ et si le coefficient de variation de $F$ est inférieur à $20 \%$ [18].

\subsection{Utilisation pratique}

La table des performances à l'effort fait ressortir les complémentarités des trois espèces. Elles travaillent dans des conditions optimales sur des plages d'efforts de traction disjointes. Ainsi, au Nord-Cameroun, le développement actuel de la traction équine complète le vide important laissé entre la traction bovine et la traction asine. Ce type de référentiel constitue une aide à la décision pour le choix d'un attelage, si l'on connaît F et PV. Prenons les exemples d'un labour superficiel et d'un travail du sol en sec au coutrier. Dans les sols ferrugineux tropicaux de la station de Garoua, un labour superficiel réalisé avec une charrue moyenne (modèle Tropic T27, de 8'') requiert une force de traction (F) comprise entre 35 et $40 \mathrm{~kg}$. Ce travail est réalisable dans des conditions d'efficacité optimales par un zébu ou un cheval (PV compris entre 225 et $450 \mathrm{~kg}$ ). Pour un âne, cet effort est trop important, ce qui justifie, dans son cas, l'emploi d'une charrue plus petite (modèle Tropic T20, de 6') ou bien d'une paire d'ânes. Le travail au coutrier dans le même type de sol, sec, requiert un effort de traction moyen de $70 \mathrm{~kg}$ [12]. L'attelage d'un cheval dans de telles conditions n'est pas envisageable. Il faudrait un cheval de plus de $450 \mathrm{~kg}$ pour fournir un tel effort de traction. Or, les races du Nord-Cameroun ne dépasse pas $300 \mathrm{~kg}$ à l'âge adulte. Pour le travail du sol en sec, le meilleur attelage est une paire de bœufs.

Le concept d'adéquation du couple animal-outil est utile pour constituer des attelages équilibrés avec un type de travail. Il n'est cependant pas suffisant pour conseiller un paysan sur le choix d'un équipement. Des critères économiques entrent en jeux dans le choix de l'animal (coût à l'achat et coût d'entretien), et des critères techniques aussi (adaptation de l'animal aux contraintes sanitaires, alimentaires, etc.). En Afrique subsaharienne, le développement de la traction animale a souvent été pénalisé par une prise en compte insuffisante des contraintes liées à l'animal. De nouvelles technologies, comme les chaînes de mesures, $s$ 'avèrent être des outils fort utiles pour combler les lacunes dans ce domaine par les investigations envisageables avec de tels appareils tant en milieu contrôlé qu'en milieu paysan.

\section{REMERCIEMENTS}

L'auteur exprime sa gratitude à $M$. Aboubakar Njoya (chef de la station de l'IRZV, de Garoua) à MM. Jean François Poulain et Seny Boukkar Lamine (responsables du Projet Garoua II), à M. Philippe Lhoste (CiradEMVT), à MM. Gérard Le Thiec, Philippe Galet et Roland Pirot (Cirad-SAR) ainsi qu'à MM. Étienne Landais, William Martin-Rosset, Gérard Matheron, Emmanuel Rossier, Jacques Thimonier, Pierre-Louis Toutain, JeanPierre Valette et Michel Vermorel (membres du comité de pilotage de la thèse de l'auteur), pour l'ensemble de leurs contributions dans la réalisation de cette étude. 


\section{RÉFÉRENCES}

[1] Betker J., Kutzbach H.D., The role of donkeys in agricultural mechanisation in Niger. Potential and limitations, in : Donkeys, mules and horses in tropical agricultural development (Fielding D., Pearson R.A., éds.), C.T.V.M., University of Edinburgh, UK (1991), 223-230.

[2] Copland J.W., Draught animal power for production, ACIAR Proceedings 10, Canberra, Australia, 1985, pp. 170.

[3] Dijkman J.T., A note of the influence of negative gradients on the energy expenditure of donkeys walking, carrying and pulling loads, in : Donkeys, mules and horses in tropical agricultural development (Fielding D., Pearson R.A., éds.) C.T.V.M., University of Edinburgh, UK, 1991, 221-222.

[4] Fielding D., Pearson R.A., Donkeys, mules and horses in tropical agricultural development, C.T.V.M., University of Edinburgh, UK (1991) pp. 336.

[5] Gallet P., Mise en place d'une chaîne de mesures et formation à son utilisation. Rapport de mission au Cameroun, Cirad-SAR, 40/92, Montpellier, France (1992) pp. 46.

[6] Goe M.R., Current status of research on animal traction, Wld. Anim. Rev. 45 (1983) 2-17

[7] G.T.Z., La traction animale en Afrique, G.T.Z., Eschborn, Germany (1982) pp. 522.

18] Hoffmann D., Nari J., Petheram R.J., Draught animals in rural development, A.C.I.A.R. Proc. 27, Canberra, Australia, 1989, pp. 347

[9] Kemp D.C., La culture attelée. Travaux récents et activités en cours, Rev. Mond. Zootech. 63 (1987) 7-14.
[10] Lawrence P.R., Pearson R.A., Factors affecting the measurements of draught force, work output and power of oxen, J. Agric. Sci. Camb. 105 (1985) 703-714.

[11] Lawrence P.R., Stibbards R.J., The energy cost of walking, carrying and pulling loads on flat surfaces by Brahman cattle and swamp buffalo, Anim. Prod. 50 (1990) 29-39.

[12] Le Thiec G., Le coutrier à traction animale. Recherche d'alternative au labour en zones sèches, C.E.E.M.A.T., Montpellier, France, 1991 , pp. 4.

[13] Le Thiec G., Agriculture africaine et traction animale, Collection Technique du Cirad, 1, Montpellier, France, 1996, pp. 362.

[14] Lhoste P., Les projets de développement de la traction animale. Les contraintes liées à l'animal et les voies d'interventions prioritaires, Cah. Rech. Dév. 21, 1989, 10-18.

[15] Scherrer J., Traction animale. Mesure de la puissance des attelages. Compte rendu de missions. 1. En Afrique, en Haute-Volta, au Sénégal et en Côte-d'Ivoire ( 9 septembre au 29 octobre 1965). 2. À Madagascar (14 janvier au 4 mars 1966), C.E.E.M.A.T., Antony, France, 1966, pp. 122

[16] Smith A.J., Recherches sur l'énergie animale. Un domaine délaissé, Rev. Mond. Zootech. 40 ( 1981 ) 43-47.

[17] Starkey P., Systèmes d'attelage et matériels à traction animale, G.A.T.E., G.T.Z., Eschborn, Germany, 1994, pp. 278.

[18] Vall E., Capacités de travail, comportement à l'effort et réponses physiologiques du zébu, de l'âne et du cheval au Nord-Cameroun, thèse de doctorat, Ensam, Montpellier, France, 1996, pp. 418. 